Journal of Computer Science 7 (1): 46-51, 2011

ISSN 1549-3636

(C) 2011 Science Publications

\title{
Reliable and Efficient Broadcasting in Asymmetric Mobile Ad Hoc Networks Using Minimized Forward Node List Algorithm
}

\author{
${ }^{1}$ Marimuthu Murugesan and ${ }^{2}$ Ammasai Krishnan \\ ${ }^{1}$ Department of Information Technology, KSR College of Engineering, \\ Tiruchengode, 637-215, India \\ ${ }^{2}$ Department of Computer Science and Engineering, KS Rangasamy College of Technology, \\ Tiruchengode, 637-215, India
}

\begin{abstract}
Problem statement: Network wide broadcasting is a fundamental operation in ad hoc networks. In broadcasting, a source node sends a message to all the other nodes in the network. Unlike in a wired network, a packet transmitted by a node in ad hoc wireless network can reach all neighbors. Therefore, the total number of transmissions (Forwarding nodes) used as the cost criterion for broadcasting. Approach: This study proposes a reliable and efficient broadcasting algorithm using minimized forward node list algorithm which uses 2-hop neighborhood information more effectively to reduce redundant transmissions in asymmetric Mobile Ad hoc networks that guarantees full delivery. Among the 1-hop neighbors of the sender, only selected forwarding nodes retransmit the broadcast message. Forwarding nodes are selected such a way that to cover the uncovered 2-hop neighbors. Results: Simulation results show that the proposed broadcasting algorithm provides high delivery ratio, low broadcast forward ratio, low overhead and minimized delay. Conclusion: In this study, reliable and efficient broadcasting algorithm in asymmetric Mobile Ad Hoc Networks using minimized forward node list algorithm has been proposed which provides low forward ratio, high delivery ratio while suppressing broadcast redundancy.
\end{abstract}

Key words:Mobile Ad Hoc Networks (MANETs), forward node, asymmetric, broadcasting, Reliable Broadcast (RB), Reliable Broadcast Protocol (RBP), dynamic network, autonomous mobile, symmetric links, Partial Dominant Pruning algorithm (PDP)

\section{INTRODUCTION}

Wireless ad hoc networks, also called Mobile Ad Hoc Networks (MANETs) are collections of autonomous mobile nodes or terminals that communicate with each other by forming a multi-hop wireless radio network. Each node in a MANET can act as both a host and a router to receive and forward packets and it can randomly move around, leave the network or switch off. Network wide broadcasting is a fundamental operation in ad hoc networks. In broadcasting, a source node sends a message to all the other nodes in the network. The advantage is that one packet can be received by all neighbors; the disadvantage is that it interferes with the sending and receiving of other transmissions, creating exposed terminal problem, that is, an outgoing transmission collides with an incoming transmission and hidden terminal problem that is, two incoming transmissions collide with each other. In general, broadcasting refers to a process of transmitting a packet so that each node in a network receives a copy of this packet. A straightforward approach for broadcasting is blind Flooding where every node in the network forwards the packet exactly once. Flooding ensures the full coverage of the entire network, that is, the Broadcast packet is guaranteed to be sent to every node in the network, providing the network is static and connected and the MAC layer of the communication channel is error-free during the broadcast process. However, flooding generates many redundant transmissions. Figure 1 shows a sample network with three nodes.

When node $\mathrm{u}$ broadcasts a packet, both nodes $\mathrm{u}$ and $\mathrm{w}$ will receive the packet. Then, $\mathrm{v}$ and $\mathrm{w}$ will rebroadcast the packet to each other. Apparently, there is much broadcast redundancy for blind flooding in this case. Transmitting the broadcast packet only by node $\mathrm{u}$ is enough for a broadcast operation. When the size of the network increases and the network becomes dense, more transmission redundancy will be introduced and these transmissions are likely to trigger considerable transmission collision and contention.

Corresponding Author: Marimuthu Murugesan, Department of Information Technology, KSR College of Engineering, Tiruchengode, 637-215, India Tel: (04288)274757 


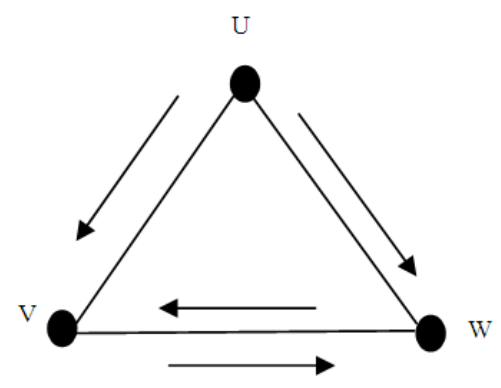

Fig 1: Redundant transmissions by blind flooding

This is a serious broadcast storm problem that finally falls down the whole network. Since MANETs suffer from transmission contention and congestion that are results of the broadcasting nature of radio transmission, it is a major challenge to provide a 'reliable broadcasting under such dynamic MANETs. This study aims to reduce broadcast redundancy by decreasing the number of the forward nodes yet still provide high delivery ratio for each broadcast packet in a dynamic environment. A subset of nodes is used to forward the broadcast message and the remaining nodes are still covered (i.e., they are adjacent to forward nodes).

Related works: Ni et al. (1999) discussed the broadcast storm problem. They also analyzed broadcast redundancy, contention and collision in blind flooding. (Siddique et al., 2007). Utilize the neighbor cache information for the AODV protocol that periodically updates it "active" neighbors for its node. The scheme introduced in the study utilizes a dynamic probabilistic broadcast coupled with the neighbor information. Thus the broadcast probability is based on the number of nodes that is kept in the neighbor cache. The scheme however does not determine whether if the neighbors in the network is proportion to the size of the network and it is does not tell of the algorithms performance against an inconsistent topology in terms of neighbor size and mobility. Peng and Lu (2000) Proposed Scalable broadcast algorithm in which a node does not rebroadcast the broadcast packet if all of its neighbors have received the packet from previous transmissions.

Ishak and Salim (2009) studied and addressed the need for efficient flood-based searching in unstructured peer-to- peer network by considering the content of query and only selecting peers that were most related to the query given. They have used minimum information to perform efficient peer selection by utilizing the past queries data and the query message. They exploited the nearest-neighbor concept on our query similarity and query hits space metrics for selecting the most relevant peers for efficient searching. Natsheh and Buragga (2010a) studied and characterized the effect of spare/dense topologies on MANET routing performance and proposes an extension for an existing routing protocol to work in such topologies.

Qayyum et al. (2002) proposed multipoint relays in which each forward node determine the status of it neighbors based on its partial 2-hop information through node coverage. MPR is source depend, that is the forward node set it dependent on the source of the broadcast .The resultant forward node set depends on many factors, such as the location of neighbors, node priority, message propagation delay and back-off delay (Wu and Dai, 2004). Proposed a generic framework for distributed broadcasting in ad hoc wireless networks. The approach is based on selecting a small subset of nodes to form a forward node set to carry out a broadcast process. The status of each node, forward or no forward is determined either by the node itself or by other nodes. Node status can be determined at different snapshots of network state along time without causing problems in broadcast coverage.

Natsheh and Buragga (2010b) improved the performance of existing MANETs routing protocols by reducing the communication overhead incurred during the route discovery process. This reduction in communication overhead is achieved by implementing a new broadcast protocol. The proposed broadcast protocol is based on the density and connectivity of the nodes and not just the number of nodes. Alagar et al. (1995) proposed a Reliable Broadcast (RB) protocol based on flooding. The protocol works as follows: The source broadcasts the message to its 1-hop neighbors. When a node receives the message; it sends an ACK back to the sender. If the message is a new one, the node retransmits the message; otherwise, it drops the message. If the sender does not receive an ACK from any of its neighbors for a predefined period, it resends the message. Garcia-Luna-Aceves and Zhang (1996). Proposed a protocol for reliable broadcasting in dynamic networks, which they called Reliable Broadcast Protocol (RBP), disseminates the same message at least once to all those network nodes that have a path to the source of the message while the message is being distributed. Khabbazian and Bhargava (2009). Proposed a sender-based flooding algorithm that can achieve local optimality by selecting the minimum number of forwarding nodes in the lowest computational time complexity $O(n \log n)$, where $\mathrm{n}$ is the number of neighbors. They showed that this optimality only holds for a subclass of senderbased algorithms. Gandhi et al. (2008) proposed a simple and distributed collision-free broadcasting algorithm for broadcasting a message and their 
algorithm and analysis extends to the case when multiple messages are broadcast from multiple sources.

Lou and Wu (2002) Proposed a Partial Dominant Pruning algorithm (PDP) to extend the DP by further reducing the number of 2-hop neighbors to be covered by 1-hop neighbors. Peng and $\mathrm{Lu}$ (2000) and Khabbazian and Bhargava (2009). Proposed a CDS based broadcast algorithm (CDSB). When a node receives a broadcast packet and determines it's forwarding nodes with lower node IDs to determine its own forwarding node set. Lou and $\mathrm{Wu}$ (2007). Have proposed a simple broadcast algorithm, called DoubleCovered Broadcast which takes advantages of broadcast redundancy to improve the delivery ratio in an environment that has rather high transmission error rate (Khabbazian and Bhargava, 2008).

With the broadcasting methods described above, they reduce the number of rebroadcasts at the expense of reach ability, longer delay; require the exchange of neighborhood information with hosts. In this study, in this study, an efficient and reliable broadcasting algorithm in Asymmetric Mobile Ad hoc Networks has been proposed which provides high delivery ratio while suppressing broadcast redundancy.

\section{MATERIALS AND METHODS}

Network model: An Ad hoc network can be modeled using a Directed Graph $\mathrm{G}=(\mathrm{V}, \mathrm{E})$. The nodes in $\mathrm{V}$ are located in a Euclidean Plane and each node $v_{i} € V$ has transmission range $\mathrm{y}_{\mathrm{i}} \in(\mathrm{y} \min , \mathrm{y} \max )$ where $\mathrm{y} \min$ is the minimum transmission range and $\mathrm{y}$ max is the maximum transmission range of a network. A directed edge $\left(v_{i}, v_{j}\right) € £$ if $d\left(v_{i}, v_{j}\right) \leq$ yi where $d\left(v_{i}, v_{j}\right)$ denote the Euclidean distance between $v_{i}$ between $v_{j}$ Such a graphs are called Directed Graphs (DG). An edge ( $v_{i}$, $\left.\mathrm{v}_{\mathrm{j}}\right)$ is bidirectional if both $\left(\mathrm{v}_{\mathrm{i}}, \mathrm{v}_{\mathrm{j}}\right)$ and $\left(\mathrm{v}_{\mathrm{j}}, \mathrm{v}_{\mathrm{i}}\right)$ are in $\mathrm{E}$. i.e., $d\left(v_{i}, v_{j}\right) \leq \min \left(y_{i}, y_{j}\right)$. Consider Fig. 2. Due to the different transmission ranges of nodes $\mathrm{u}, \mathrm{v}, \mathrm{w}$, if there is an asymmetric link ( $\mathrm{u}, \mathrm{v})$ from node $\mathrm{u}$ to $\mathrm{v}$ and symmetric links between $v$ and $w$ and between $w$ and $u$, $\mathrm{v}$ realizes the asymmetric link ( $\mathrm{u}, \mathrm{v})$ if $\mathrm{v}$ receives the HELLO message from $u$ with u' $s$ 1-hop neighbor set and finds itself not in 1-hop neighbor set, $\mathrm{v}$ starts a local broadcast REQ to find $u$. Intermediate node $w$ attaches ID and forwards the REQ. When $u$ receives the $\mathrm{REQ}$, it recognized the asymmetric link (u, v) and builds the feedback path ( $\mathrm{v}$, wand $\mathrm{u}$ ) and informs $\mathrm{v}$ of the feedback path.

Minimized forward node list algorithm: Let $\mathrm{S}$ be the node that determine its forwarding node list $\mathrm{FNL}(\mathrm{S})$.
$\mathrm{N}(\mathrm{S})$ represents the 1-hop neighbor list of $\mathrm{S}$ (including $\mathrm{S}$ ). $\mathrm{N}_{2}(\mathrm{~S})$ represents the 2-hop neighbor list of $\mathrm{S}$ (i. e, the set of nodes that are within two hops from $S$ ).

Clearly $\{\mathrm{S}\} \subseteq \mathrm{N}(\mathrm{S}) \subseteq \mathrm{N}_{2}(\mathrm{~S})$

If $v € N(S)$ then $N(v) \subseteq N_{2}(S)$

The node $\mathrm{S}$ of the broadcast operation uses the following algorithm to determine its forwarding node list:

- $\quad$ Node $S$ computes $X=N(S)-S$ and $\mathrm{UCL}(\mathrm{S})=$ $\left.\mathrm{N}_{2}(\mathrm{~S})\right)-\mathrm{N}(\mathrm{S})$ and $\mathrm{FNL}(\mathrm{S})=\varphi$

- First select those 1-hop neighbor nodes in $\mathrm{X}$ as forward nodes which are the only neighbor of some node in UCL(S). Add these 1-hop neighbor nodes to the $\operatorname{FNL}(\mathrm{S})$ and remove from $\mathrm{X}$. Also remove the 2-hop neighbors which are covered by the above 1-hop neighbors from UCL(S)

- Find $w($ in $X)$ with maximum effective neighbor degree using deg (w) which consists of nodes that is in both $\mathrm{N}(\mathrm{w})$ and UCL $(\mathrm{S})$

- $\operatorname{FNL}(\mathrm{S})=\operatorname{FNL}(\mathrm{S}) \mu\{\mathrm{w}\}, \mathrm{UCL}(\mathrm{S})=\mathrm{UCL}(\mathrm{S})-\mathrm{N}$ (w) and $X=X-\{w\} \backslash$

- Repeat step 3 and 4 until UCL(S) becomes empty

Figure 3 shows a sample network of 11 nodes with source node 1 . Neighborhood information of each node is shown in Table 1.

Table 1: Neighborhood information

\begin{tabular}{lll}
\hline $\mathrm{V}$ & $\mathrm{N}(\mathrm{v})$ & $\mathrm{N}_{2}(\mathrm{v})-\mathrm{N}(\mathrm{v})$ \\
1 & $1,2,3,4,5,6$ & $7,8,9,10$ \\
2 & $1,2,3,7,8,9$ & $4,5,6,11$ \\
3 & $2,3,7,8$ & 1,9 \\
4 & $1,2,4,9$ & $3,5,6,7,8$ \\
5 & 10 & $1,3,4$ \\
6 & $1,3,5,6$ & $2,4,7,8,10$ \\
7 & $2,3,7,8$ & 1,9 \\
8 & $2,7,8,11$ & 1,9 \\
9 & 4,9 & $\mathrm{~S} 1,2,3,7$ \\
10 & 4,10 & $1,2,9$ \\
\hline
\end{tabular}

Source: (Node1) is covered

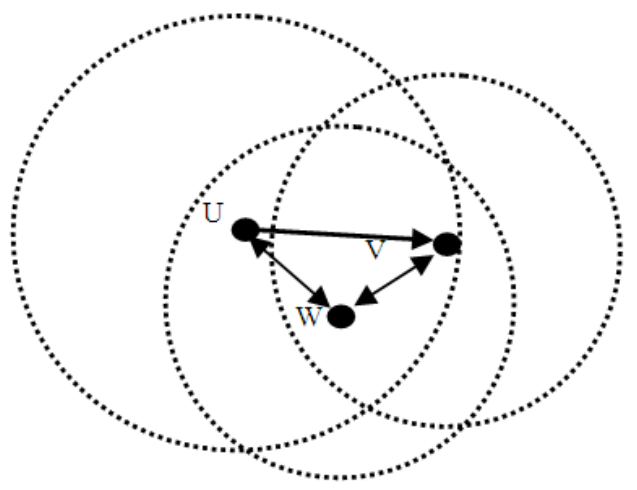

Fig. 2: Asymmetric mobile ad hoc network 


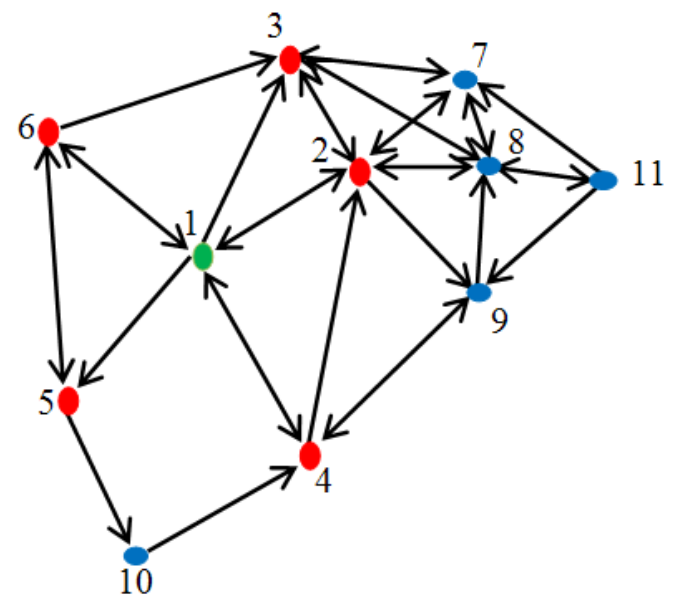

Fig. 3: A sample asymmetric Manet

For the MFNL algorithm, nodes in $\mathrm{N}$ (1) will directly receive the packet. The uncovered list of node 1 is shown as UNCL $(1)=\mathrm{N}_{2}(\mathrm{v})-\mathrm{N}(\mathrm{v})=\{7,8,9,10\}$. The node 1 selects its forwarding node with the maximum degree, so the forwarding node list for node 1 is FNCL $(1)=\{2,4,5$,$\} . The forwarding node v$ of node $u$ selects its forwarding node by $\mathrm{N}(\mathrm{v})-\mathrm{N}(\mathrm{u})-\mathrm{N}(\mathrm{FNL}(\mathrm{u}))$, like this the uncovered list of source(node1) is covered.

Reliable and Efficient Broadcast Algorithm (REBA): A reliable broadcast operation requires the packet to be received by all the nodes in the network. But the interference of the transmission of neighbors and the movement of the nodes may cause the failure of some nodes to receive the packet. Therefore, the sender needs to retransmit the packet to increase the delivery ratio of the transmission.

The proposed reliable broadcast algorithm works as follows:

- When a source broadcast a packet, it selects some neighbors from its 1-hop neighbor set that form its forwarding node list to cover its 2-hop node set

- After the forward nodes receive the new broadcast packet, each forward node records the packet, computes its forward nodes and rebroadcasts the packet

- The retransmissions of the forwarding nodes are overheard by the sender as the acknowledgement of the reception of the packet. The non forwarding 1-hop neighbors of the sender only explicitly acknowledge the receipt of the packet

- The sender waits for a predefined duration to overhear the rebroadcast from its forwarding nodes and to receive ACKs from its non forwarding nodes. If the sender fails to detect all its forwarding nodes retransmission and does not receive all non forwarding nodes ACKs during this duration, it assumes that a transmission failure has occurred for this broadcast and the packet

- If the sender fails to receive ACKs from all its non forwarding nodes and fails to detect all forwarding nodes retransmission for a threshold number of times, the sender assumes the nodes that do not reply are out of its transmission range and stops further attempts

- If the node that misses the packet is a non-forward node, then the missed packet does not cause other missing propagations in the network. On the other hand, if it is a forward node that misses the broadcast packet, this miss may propagate through the network since the neighbors of this forward node will also miss the packet. So when a sender fails to detect retransmission of a forward node after maximum number of retries, it reselects alternative forward node to cover the set which is supposed to be covered by it

Theoretical Model for the proposed Broadcasting Algorithm: The proposed broadcasting algorithm achieves $100 \%$ delivery ratio if and only if for each node $S$ the neighbor's area of $S$ is covered by $\mathrm{FNL}(\mathrm{S})$.

Proof sufficient condition: Suppose for each node $s$ the two-hop neighbor's of $\mathrm{S}$ is covered by $\mathrm{FNL}(\mathrm{S})$ ). It is required to prove that the proposed broadcasting algorithm attain $100 \%$ deliverability. For each transmission node $S$, since all two-hop neighbors of $S$ are neighbors of S's one-hop neighbors, they are sure to be covered by nodes in FNL(S). Thus, all nodes that are two-hop away from the source $S$ are sure to be covered by $\mathrm{FNL}(\mathrm{S})$. Notice that S's 3-hop neighbors are neighbors of S's 2-hop neighbors. There must exist some transmission nodes in FNL(S), such that S's 3hop neighbors are 2-hop neighbors of these transmission nodes. Thus, S's 3-hop neighbors are sure to be covered by forwarding sets of these transmission nodes. Nodes that are 4-hop and more from the source can be proved in the similar way. Therefore, the broadcasting message will be forwarded hop by hop throughout the whole network.

Necessary condition: Suppose the proposed algorithm achieves $100 \%$ delivery ratio. Let $\mathrm{FNL}(\mathrm{S})$ denote the set of forwarding nodes of $\mathrm{S}$ that is computed by the proposed forward node list algorithm MFNL. It is needed to prove that for each node $S$ the neighbors of $S$ are covered by MFNL(S). We prove it by contradiction. 
Let us consider a kind of networks where all nodes are within the transmission range of a central node, denoted by $\mathrm{S}$. That is, any network in this category consists of a central node and its neighbors, shown in Fig. 3. Suppose the proposed algorithm does not guarantee that for each node $\mathrm{S}$ the two-hop neighbor's $\mathrm{S}$ is covered by MFNL(S). There must exist such a network as shown in Fig. 4 and the two-hop neighbor of $\mathrm{S}$ is not fully covered by MFNL(S). Since $\mathrm{F}_{\min }(\mathrm{S})$ is the smallest forwarding set that overs the two-hop neighbor's of $\mathrm{S}$, then $\mathrm{F}_{\min }(\mathrm{S}) \not \subset \mathrm{FNL}(\mathrm{S})$. In other words, there exists node $\mathrm{u} \in \mathrm{F}_{\min }(\mathrm{S})$ and $\mathrm{u} \notin \operatorname{MFNL}(\mathrm{S})$ ). Notice that all nodes in $F_{\min }(S)$ are sure to contribute to cover the two hop neighbor's of $\mathrm{S}$ (if not, it can be removed from $\mathrm{F}_{\min }(\mathrm{s})$ ). On the other hand, since there is no other nodes outside coverage disk of $s$, node $v$ can neither be covered by forwarding set of other nodes. That is, node $v$ will eventually miss the flooding message. It contradicts the assumption that flooding scheme $A$ achieves 100\% deliverability. The above proof tells that the sufficient and necessary condition of $100 \%$ deliverability for the proposed algorithm is that for each node $\mathrm{S}$, the neighbor's area of S should be covered b y MFNL(S). Otherwise, some nodes in the network may miss the broadcasting message.

\section{RESULTS}

Simulations were carried out using ns-2 simulator which is a well known packet level simulator, toevaluate the proposed broadcasting algorithm. The delivery ratio, forwarding ratio, overhead and delay of the proposed broadcasting algorithm REBA is compared with Blind blooding, Reliable broadcasting and Double covered broadcasting in Fig. 5-8. In simulations, the number of nodes are changed from 20 to 100 . Simulation parameters are listed in Table 2.

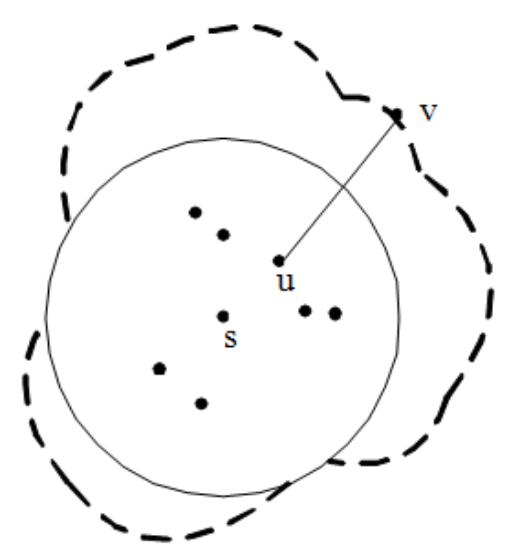

Fig. 4: Example of neighbor's area of $s$
Table 2: Simulation parameters

\begin{tabular}{ll}
\hline Parameter & Value \\
\hline Simulator & NS-2 (version 2.31) \\
Network area & $900 \times 900 \mathrm{~m}^{2}$ \\
Transmission range & $250 \mathrm{~m}-400 \mathrm{~m}$ \\
MAC layer & IEEE 802.11 \\
Data packet size & 64 bytes \\
Bandwidth & $2 \mathrm{Mbps}$ \\
Simulation time & $100 \mathrm{~s}$ \\
Number of trials & 10 \\
\hline
\end{tabular}

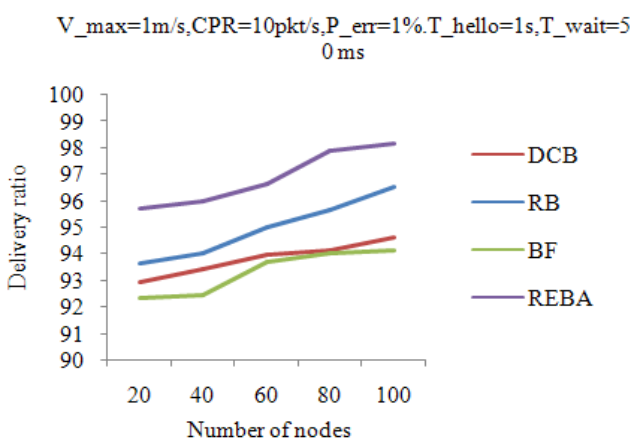

Fig. 5: Delivery Ratio-Sensitivity to mobility of the node

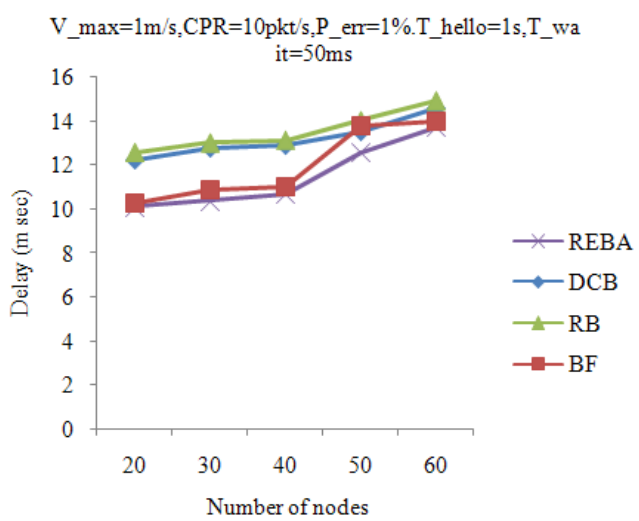

Fig. 6: Delay-Sensitivity to mobility of the node

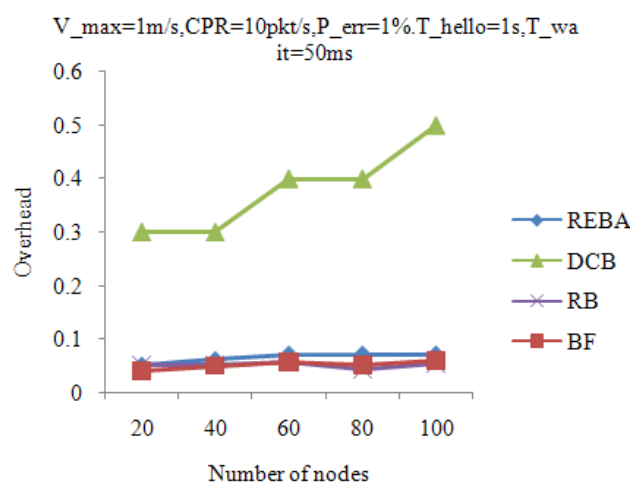

Fig. 7: Overhead-Sensitivity to mobility of the node 


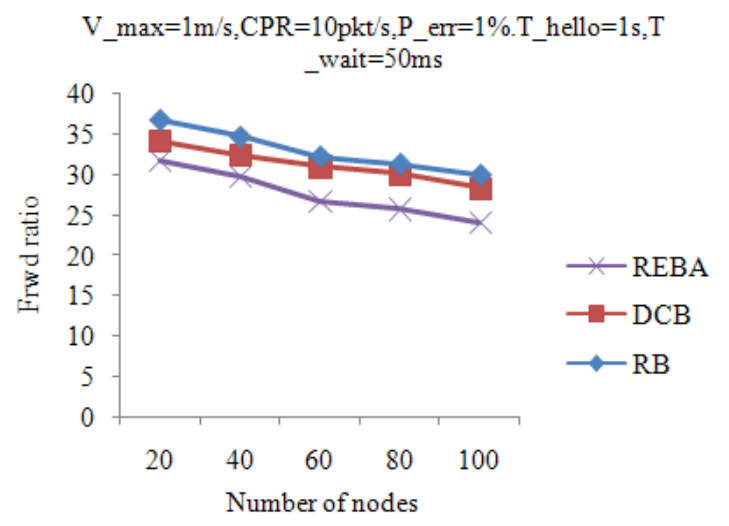

Fig. 8: Forward Ratio-Sensitivity to mobility of the node

\section{DISCUSSION}

The proposed broadcasting algorithm provides good delivery ratio, low forwarding ratio, low overhead and less delay compared to other well known broadcasting algorithms like blind flooding, reliable broadcasting and double covered broadcasting.

\section{CONCLUSION}

In this study, a reliable and efficient broadcasting $t$ algorithm in Asymmetric Mobile Ad hoc Networks has been proposed which provides low forward delivery ratio ,high delivery ration low overhead and less delay while suppressing broadcast redundancy. This is achieved by only requiring some selected forwarding nodes among the sender's 1-hop neighbor set to forward the packet. The directed DGs can be used to model wireless ad hoc networks, where nodes have different transmission ranges. The simulation results show that the proposed broadcast algorithm low forwarding ratio.

\section{REFERENCES}

Alagar, S., S. Venkatesan and J. Cleveland, 1995 Reliable broadcast in mobile wireless networks. Proceeding of the Military Comm. Conference, pp: 236-240. DOI: 1109/ MILCOM. 1995. 483305

Gandhi, R., A. Mishra and S. Parthasarathy, 2008. Minimizing broadcast latency and redundancy in ad hoc networks. IEEE/ACM Trans. Network., 16. DOI: 10.1109/TNET.2007.905588

Ishak, I. and N. Salim, 2009. Selective flooding based on relevant nearest-neighbor using query feedback and similarity across unstructured peer-to-peer networks. J. Comput. Sci., 184-190. ISSN: 1549-3636
Garcia-Luna-Aceves, J.J. and Y.X. Zhang, 1996. Reliable broadcasting in dynamic network. Proceeding 1996 IEEE International Conference Communication, pp: 1630-1634. DOI: 10.1109/ICC.1996.535192

Natsheh, E. and K. Buragga, 2010a. Nodes density and broadcast management in heterogeneous environments of mobile ad-hoc networks. J. Comput. Sci., 6: 312-319. ISSN 1549-3636

Khabbazian, M. and V.K. Bhargava, 2009. Efficient broadcasting in mobile ad hoc networks. IEEE Trans. Mobile Comput., 8: 231-245. ieeecomputersociety.org/10.1109/TMC.2008.94

Natsheh, E. and K. Buragga, 2010b. Density based routing algorithm for spare/dense topologies in wireless mobile ad-hoc networks. Am. J. Eng. Applied Sci., 3: 312-319.

Lou, W. and J. Wu, 2002. On reducing broadcast redundancy in ad hoc wireless networks, 1: 111122. DOI: 10.1109/TMC.2002.1038347

Khabbazian, M. and V.K. Bhargava, 2008. Localized broadcasting with guaranteed delivery and bounded transmission redundancy. IEEE Trans. Comput., 57: 1072-1086. DOI: 10.1109/TC.2008.51

Lou, W. and J. Wu, 2007. Toward broadcast reliability in mobile ad hoc networks with double coverage. IEEE Trans. Mobile Comput., 2: 148-163. DOI: TMC-0218-0705

Ni, S., Y. Tseng, Y. Chen and J. Sheu, 1999. The broadcast storm problem in mobile ad hoc networks. Proceeding of the ACM/IEEE International Conference on Mobile Computing and Networking, Aug. 15-19, AMC Press, Seattle, Washington, United States, pp: 151-162. DOI: $10.1145 / 313451.313525$

Peng, W. and X. Lu, 2000. On the reduction of broadcast redundancy in mobile ad hoc networks. Proceeding MobiHoc Conference, pp: 129-130. DOI: 10.1109/ MOBHOC.2000.869221

Qayyum, A., L. Viennot and A. Laouiti, 2002. Multipoint relaying for flooding broadcast message in mobile wireless networks. Proceeding of the 35th Hawaii International Conference System Science, pp: 3898-3907. DOI: 10.1109/HICSS.2002.994521

Siddique, A., A.M. Hanashi, I. Awan and M. Woodward, 2007. Performance evaluation of dynamic probabilistic flooding using local density information in MANETs. Network-Based Inform. Syst., 4658: 288-297. DOI: 10.1007/978-3-54074573-0

Wu, J. and F. Dai, 2004. A generic distributed broadcast scheme in ad hoc wireless networks. IEEE Trans. Comput., 53: 1343-1354. DOI: 10.1109/TC.2004.69 